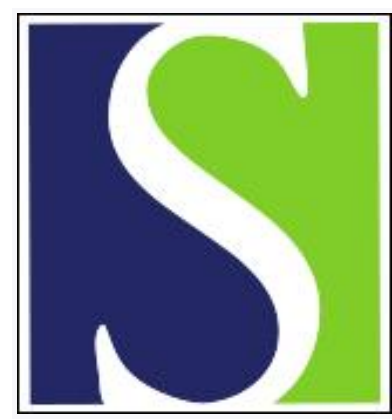

Scand J Work Environ Health 1997;23(2):81-82

https://doi.org/10.5271/sjweh.184

Issue date: Apr 1997

\title{
Asbestos -- a continuing concern
}

by Karjalainen $\mathrm{A}$

The following article refers to this text: 2003;29(1):64-70

This article in PubMed: www.ncbi.nlm.nih.gov/pubmed/9167230

\section{(c) (i)}




\section{Asbestos - a continuing concern}

Exposure to asbestos increases the risk of lung cancer, mesothelioma, pulmonary fibrosis (asbestosis), and various pleural diseases. Despite extensive research on the topic, the mechanisms of asbestos carcinogenicity are only partly understood. Two implications of this lack of knowledge are touched upon in this issue. Merler and his colleagues have reviewed the modalities of surveillance and health intervention for asbestos-exposed workers. Apart from the elimination of exposure and the cessation of smoking, very few evidence-based tools seem to be available for reducing cancer mortality or promoting the health of exposed workers. In the other review Hillerdal \& Henderson summarize the extensive literature dealing with the question of whether asbestos fibers as such, or asbestosis, is the reason for the increased risk of lung cancer. The question has 2 major practical implications: the strategies of primary prevention and the compensation for lung cancer among asbestos-exposed workers.

The epidemiologic evidence of a causal relationship between asbestos exposure and mesothelioma is convincing and, despite the long latency time, some examples of successful prevention already exist. The incidence of mesothelioma has decreased in certain areas in the 1990s, but not everywhere $(1,2)$. In addition the statistics of the Finnish Cancer Registry indicate a plateau or a decline in the 1990s for men under 55 years of age and for women, while the incidence is still rising for men over 65 years of age (3). Up to 1995 the highest annual number of cases of mesothelioma was 57 in 1989. Meanwhile, the reporting of work-related cases has reached a high level, about $80 \%$ of all mesotheliomas in men.

Preliminary data indicate possible modifying genetic and exogenous determinants for the asbestosassociated risk of mesothelioma $(4,5)$. Such findings may hopefully improve our understanding of the carcinogenic process, but eliminating the hazardous asbestos exposure remains the only effective means of prevention. Asbestos-related lung cancer is numerically even more important than mesothelioma. Hillerdal \& Henderson point out that lung cancer is a common disease and even a low relative risk, if common, will result in a large number of lung cancers. The national policies vary in respect to whether the scientific evidence is considered to justify a total ban or the limited use of asbestos. The Netherlands, Sweden, Norway, and Denmark were the first countries to implement a total ban. In the 1990s the same decision was made in Germany, Finland, and, quite recently, France. Meanwhile, the use of chrysotile asbestos is increasing in Asia and many other regions. It is to be expected that full control of exposure levels will be a difficult, if not impossible task in these societies.

Surveillance and periodic medical examinations have an obvious social role in detecting and monitoring asbestos-associated occupational diseases, albeit their health benefits have not been evaluated. The risk of asbestos-associated diseases among exposed workers can be reduced by the elimination of asbestos exposure and the cessation of smoking, although Merler \& his colleagues point out that more could perhaps be known, and better results achieved, if ongoing programs were integrated. Screening for lung cancer with $X$ rays or sputum cytology is not considered beneficial. Nor do chemoprevention trials among asbestos-exposed workers so far indicate evidence of protection. In lung cancer screening studies, the number of lung cancer deaths in the screening group has been at least as high as in the reference group, and some chemoprevention trials have been interrupted due to adverse effects. There is a need for new earlydiagnostic tools or new chemopreventive agents, but their application must be based on controlled trials.

An important task in the surveillance of exposed workers is to provide them with adequate information on the health risks of asbestos. The right to know should be a self-evident principle. Motivation to avoid further exposure and to quit smoking is also enhanced by this information. On the other hand, one should not inspire fear in those who have been exposed. The provision of balanced and adequate information is a real challenge for those involved in the follow-up of exposed workers. In a Finnish screening campaign among construction and shipyard workers, the information given during the screening activities reduced the concern of the personal health risk of past exposure for $15 \%$ of those who were worried (6). For the rest, the concern increased or remained unchanged. The information aspect should not be forgotten when the details of future surveillance activities or health intervention is planned for exposed workers.

Exposure intensity and the presence of pulmonary fibrosis are closely related, and epidemiologic studies will probably never lead to a scientific consensus on whether the fiber itself, the consequent 
fibrosis, or its progression is the reason for the increased risk of lung cancer. A better mechanistic understanding may reveal an independent role for each of these. Meanwhile, the compensation for lung cancer remains a controversial issue, from the point of view of both science and practice. Chest $X$ rays, high resolution computed tomography (HRCT), and histology possess different sensitivities in detecting pulmonary fibrosis, and a medical differential diagnosis between (mild) asbestosis and other diffuse pulmonary fibrosis is not always simple. The sensitivity of CT instruments is continuously increasing, and there are no studies that have compared the occurrence of the various HRCT findings between asbestos-exposed and unexposed persons. If cumulative exposure and the relative risk of lung cancer, and not just the presence of fibrosis, are accepted as the basis for compensation, practical problems may arise from relatively low exposure and risks. When legal compensation is concerned, apportionment according to the estimated etiologic fractions of asbestos, tobacco smoking, and other lung carcinogens at the individual level is a justifiable solution. On the other hand, if the compensation for occupational diseases is integrated into the social security system, a dichotomous concept is reasonable. A system in which a varying part of an individual's social security comes from 1 source and the rest from another may cause problems. A relative risk of 2 is commonly used as a cut point for a dichotomous system.

While the administrative basis for compensation is a political question, an abundance of scientific data is available to help to develop the actual criteria. A thorough exposure interview is often the best estimate of exposure. Reliable complementary information on the intensity of exposure and the consequent relative risk of lung cancer can be achieved by lung and bronchoalveolar lavage asbestos measurements, whereas recent Scandinavian studies confirm that the presence of pleural plaques as such indicate only a rather small relative risk of lung cancer, if any $(7,8)$. In the Finnish population, the risk of lung cancer was found to be 17-fold for asbestos sprayers, about 3 -fold for insulators and asbestos quarry workers, 1.5 -fold for plumbers, and 1.3-fold for construction carpenters. The exposure gradient behind this risk pattern was clearly reflected by the asbestos concentrations in pulmonary and bronchoalveolar lavage samples of workers in these occupations and unexposed referents. Finnish construction workers with long-term exposure to asbestos and pleural plaques as an occupational disease had a 1.2-fold risk of lung cancer.

The surveillance of former asbestos workers and the compensation for asbestos-related cancers remain long-standing social challenges. Even though the occurrence of cancers may already have reached the maximum in some areas, the decline is unlikely to be rapid. This situation is unforgivable, as the basic knowledge for effective primary prevention was available already in the mid-1960s. Although the minimization of future exposures is the most important task, the concern for the health of past asbestos workers is an undeniable social responsibility. It should, however, not create the illusion that hazardous asbestos exposure is a past problem in modern societies. The quality of asbestos demolition work is a continuous problem, and a recent survey in Finland found occasional high fiber concentrations even inside personal protectors during asbestos removal work. There is a need for continuous education and a more systematic health surveillance of asbestos demolition workers.

\title{
References
}

1. Damhuis RAM, Planteydt HT. Mesothelioma [letter]. Lancet 1995;345:1233 - 4.

2. Peto J, Hodgson JT, Matthews FE, Jones JR. Continuing increase in mesothelioma mortality in Britain. Lancet 1995:345:535-9.

3. Karjalainen A, Pukkala E, Mattson K, Tammilehto L, Vainio H. Trends in mesothelioma incidence and occupational mesotheliomas in Finland 1960-95. Scand J Work Environ Health. In press.

4. Hirvonen A, Saarikoski ST, Linnainmaa K, Koskinen K, Husgafvel-Pursiainen K, Mattson K, et al. Gluthatione $\mathrm{S}$-transferase and $\mathrm{N}$-acetyltransferase genotypes and asbestos-associated pulmonary disorders. JNCI 1996;88:1853 -6.

5. Pepper C, Jasani B, Navabi H, Wynford-Thomas D, Gibbs AR. Simian virus 40 large T antigen (SV40LTAg) primer specific DNA amplification in human pleural mesothelioma tissue. Thorax 1996;51:1074 6 .

6. Koskinen K, Rinne J-P, Kivekäs J, Zitting A, Tossavainen A, Roto P, et al. Asbestisairauksien seulontatutkimus 1990—92 [Screening of asbestos-induced diseases 1990 - 92]. Helsinki: Finnish Institute of Occupational Health, 1993.

7. Hillerdal G. Pleural plaques and risk for bronchial carcinoma and mesothelioma: a prospective study. Chest 1994;105:14450 .

8. Waage H, Johnson E, Hilt B, Langård S. Asbestosis and pleural changes as risk factors for asbestos-induced lung cancer. Int J Occup Med Toxicol 1994;3:319—27.

\author{
Antti Karjalainen \\ Finnish Institute of Occupational Health \\ Topeliuksenkatu 41 a $\mathrm{A}$ \\ FIN-00250 Helsinki Finland
}

